\title{
Intra-arterial thrombectomy in elderly patients: assessment of arterial tortuosity in determining successful procedure
}

\author{
Su Min Kye, Jun Hyong Ahn, In Bok Chang, Joon Ho Song, Ji Hee Kim, Heui Seung Lee, Jae Keun Oh \\ Department of Neurosurgery, Hallym University Sacred Heart Hospital, College of Medicine, Hallym University, Anyang, Korea
}

Objective: Intra-arterial thrombectomy (IAT) is the mainstay of initial treatment for intracranial large-vessel occlusion. However, arterial tortuosity is associated with procedural failure and unfavorable outcomes in older patients.

Methods: We reviewed patients with ischemic stroke ( $>75$ years) treated with IAT and analyzed procedure-related complications and arterial tortuosity based on pre-procedural computed tomography angiography.

Results: Of the 29 patients, 7 had distal internal carotid artery (ICA) occlusion, 18 had middle cerebral artery (MCA) occlusion, 3 had anterior cerebral artery and MCA occlusion, and one had distal ICA and MCA occlusion. Significant arterial tortuosity was observed in 9 patients (31.0\%). The degree of recanalization, as assessed by the modified thrombolysis in cerebral infarction grade, did not differ according to significant arterial tortuosity $(P=0.55)$. Post-procedural subarachnoid hemorrhage was observed in 17 of 29 patients $(58.6 \%)$ and there was no significant association with significant arterial tortuosity. The puncture to recanalization time was not significantly different according to the presence of significant arterial tortuosity ( $51.6 \pm 22$ minutes vs. $53 \pm 43.9$ minutes; $P=0.93)$. Additionally, the number of thrombus retrieval trials during the procedure did not show a significant relationship with arterial tortuosity $(2.4 \pm 1.2$ times vs. $2.6 \pm 2.5$ times; $P=0.86)$.

Conclusion: IAT can be performed in patients aged $>75$ years. Arterial tortuosity was not significantly associated with successful recanalization or post-procedural complications. Therefore, whether to perform IAT in older patients should not be determined only by arterial tortuosity.

Keywords: Elderly patients; Carotid arteries; Stroke; Thrombectomy; Tortuosity

\section{Introduction}

Intra-arterial thrombectomy (IAT) has become the mainstay of treatment for ischemic stroke caused by large artery occlusion $[1,2]$. However, IAT in older patients should be carefully considered due to uncertain clinical outcomes despite the risk of procedure-related complications $[3,4]$. Vascular tortuosity and longer procedure time have been suggested as factors associated with

Received: August 6, 2021

Revised: October 8, 2021

Accepted: October 14, 2021

Corresponding Author: Heui Seung Lee, MD

Department of Neurosurgery, Hallym University Sacred Heart Hospital, College of Medicine, Hallym University, 22 Gwanpyeong-ro 170beon-gil, Dongan-gu, Anyang 14068, Korea

Tel: +82-31-380-1500; Fax: +82-31-380-4118;

E-mail:antanatia@gmail.com unfavorable outcomes in older patients who underwent IAT $[5,6]$.

Previous studies have reported a higher rate of post-procedural hemorrhage and subsequent mortality in older patients with acute ischemic stroke and have suggested that IAT does not have a definitive benefit $[7,8]$. Meanwhile, technical improvements as well as advances in endovascular devices should be considered when deciding on IAT for older patients.

In this study, we reviewed clinical outcomes after IAT in older patients and evaluated the factors associated with unfavorable outcomes of IAT. Therefore, we also evaluated whether it is reasonable to restrict IA thrombectomy in older patients based on factors associated with the vascular structure, such as arterial tortuosity. 


\section{Material and Method}

\section{Patients and data collection}

Patients over 75 years of age who underwent IAT for ischemic stroke due to anterior cerebral circulation occlusion from were retrospectively reviewed. Patients arriving at the hospital within 4.5 hours were treated with intravenous tissue plasminogen activator in the absence of contraindications. Patients with a contraindication of intravenous thrombolysis were directly treated with IAT within 18 hours from the patients' last normal time.

A balloon guiding catheter was used and intermediate catheter was placed in the petrous segment of the internal carotid artery (ICA). The Solitaire FR (flow restoration; Covidien, Irvine, CA, USA) was embedded at the occlusion site with a minimum embedding time of 3 minutes and retrieval of the stent was performed simultaneously with the suction of the intermediate catheter.

Patients who underwent emergent carotid stenting for proximal ICA occlusion and IAT for posterior circulation occlusion were excluded. The grade of recanalization was estimated using a modified thrombolysis in cerebral infarction (mTICI) score. This study was approved by our institutional review board.

\section{Definition of arterial tortuosity}

We define arterial tortuosity as ICA dolichoectasia and common carotid artery (CCA) takeoff angle, which was previously published by Knox et al. [9] Significant ICA dolichoectasia is defined as $>60^{\circ}$ in 2 segments kinking of proximal ICA (Fig. 1A), and the CCA takeoff angle means measurement of the left CCA on computed tomography angiography with the angle of the spine as a reference (Fig. 1B).

\section{Definition of recanalization}

We estimate recanalization using the mTICI score, as grade 0 means that there was no perfusion after the IAT procedure; grade 1 , as angiography showed penetration with minimal perfusion; grade 2, partial perfusion after thrombectomy; and grade 3, complete perfusion after the intervention.

\section{Statistical analysis}

IBM SPSS ver. 22.0 (IBM Corp., Armonk, NY, USA) was used for all statistical analyzes. Data normality was assessed using the Kolmogorov-Smirnov test. The independent $\mathrm{t}$-test was used to evaluate the differences between continuous variables. The chisquare test was performed to determine the differences between categorical variables. Statistical significance was set at $\mathrm{P}<0.05$.

\section{Results}

In total, 29 patients were analyzed. The mean age of the patients was $81.1 \pm 4.99$ years and the median age was 81 years (interquartile range, $76-84$ ). Female patients comprised $62.1 \%$ of the enrolled patients $(\mathrm{n}=18)$. The underlying diseases of the patients

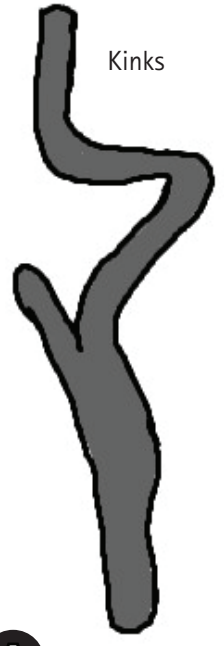

A
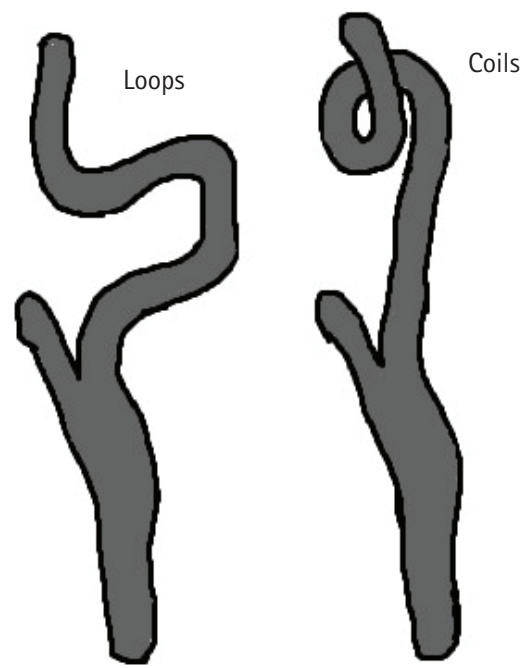

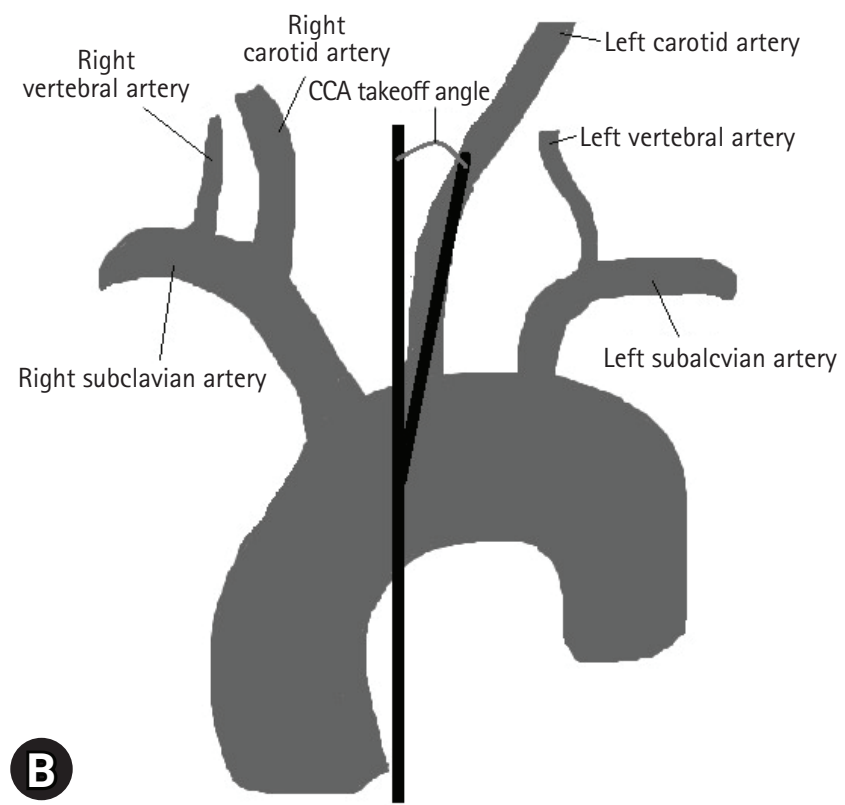

Fig. 1. (A) Significant internal carotid artery (ICA) dolichoectasia was defined as kinking of the proximal ICA of $>60^{\circ}$ in 2 segments. (B) The common carotid artery (CCA) takeoff angle denoted a measurement of the left CCA on computed tomography angiography with the angle of the spine as a reference. 
were diabetes mellitus ( $n=6,20.7 \%)$, hypertension $(n=20$, $69.0 \%)$, and atrial fibrillation $(\mathrm{n}=16,55.2 \%)$. Ten patients had already been treated with antiplatelet anticoagulants. The median values of the initial National Institute of Health Stroke Scale (NIHSS) score and initial Alberta Stroke Programmed Early Computed Tomography Score (ASPECTS) were 16 and 8, respectively (Table 1).

Occlusion occurred on the right side in 18 patients and on the left side in 16 patients. There were 7 cases of distal ICA occlusion, 18 cases of middle cerebral artery (MCA) occlusion, and 4 cases of tandem lesions (Table 2).

As an aspect of the procedure, there were 3 cases in which the procedures were ended only for aspiration, the rest of the cases, the

Table 1. Clinical and demographic information of 29 patients who underwent intra-arterial thrombectomy due to anterior circulation occlusion

\begin{tabular}{lc}
\hline Variable & Value $(\mathrm{n}=29)$ \\
\hline Age (yr) & \\
$\quad$ Mean \pm SD (range) & $81.1 \pm 4.99(75-91)$ \\
$\quad$ Median (IQR) & $81(76-84)$ \\
Female/male (\%) & $18(62.1) / 11(37.9)$ \\
Diabetes mellitus (\%) & $6(20.7)$ \\
Hypertension (\%) & $20(69.0)$ \\
Atrial fibrillation (\%) & $16(55.2)$ \\
Anticoagulant or antiplatelet medication (\%) & $10(34.5)$ \\
Initial NIHSS (median, IQR) & $16(14-18)$ \\
Initial ASPECTS (median, IOR) & $8(7-9)$ \\
\hline
\end{tabular}

SD, standard deviation; IQR, interquartile range; NIHSS, National Institute of Health Stroke Scale; ASPECTS, Alberta Stroke Programmed Early Computed Tomography Score.

Table 2. Location of occlusion sites

\begin{tabular}{lc}
\hline Variable & Value \\
\hline Right side/left side & $18 / 16$ \\
Solitary lesion & 7 \\
Distal ICA & 1 \\
I occlusion & 2 \\
L occlusion & 4 \\
T occlusion & 18 \\
MCA & 15 \\
M1 & 3 \\
M2 superior & 0 \\
M2 inferior & 4 \\
Tandem lesion & 1 \\
Proximal ICA, MCA & 3 \\
MCA, ACA & 3 \\
\hline
\end{tabular}

ICA, internal carotid artery; MCA, middle cerebral artery; $A C A$, anterior cerebral artery. stent retriever, and aspiration were performed. The mean time from puncture to recanalization was 52.1 minutes. The mean passage try for thrombus retrieval was 2.5 times and there were $11 \mathrm{pa}-$ tients who needed more than 3 times thrombus passage (Table 3 ).

\section{Angiographic outcome}

Twenty-two patients showed recanalization of the artery more than the mTICI score $2 b$, and 7 patients showed recanalization with mTICI score 0-2a. After the procedure, 17 patients had subarachnoid hemorrhage (SAH), 13 patients had distal embolism, and 7 patients had parenchymal intracerebral hemorrhage (ICH). Twenty patients (69.0\%) had a modified Rankin scale (mRS) score of more than 3 points at discharge (Table 4 ).

For the mRS score $\geq 3$ points at the time of patient discharge, the factors for poor prognosis that showed correlation or showed correlation trend were initial NIHSS score $(\mathrm{P}=0.08)$, initial ASPECTS $(\mathrm{P}<0.001)$, mTICI score $(\mathrm{P}=0.06)$; there was no correlation between poor prognosis and post-procedure $\mathrm{SAH}$, distal embolism, parenchymal ICH (Table 5).

Table 3. Information about the procedures

\begin{tabular}{lc}
\hline Variable & Value $(n=29)$ \\
\hline Aspiration only & $3(10.3)$ \\
Stent retriever+aspiration & $26(89.7)$ \\
Puncture to recanalization $(\mathrm{min})$ & $52.1 \pm 29.7$ \\
No. of passes & $2.5 / 2(1-8)$ \\
$\quad$ No. of passes $<3(\%)$ & $18(62.1)$ \\
No. of passes $\geq 3(\%)$ & $11(37.9)$
\end{tabular}

Values are presented as number (\%), mean \pm standard deviation, or mean/ median (range).

Table 4. Angiographic and patients' outcomes

\begin{tabular}{lr}
\hline Variable & \multicolumn{1}{c}{ Value } \\
\hline Final mTICl score (\%) & $7(24.1)$ \\
$0-2 a$ & $22(75.9)$ \\
$2 b-3$ & \\
Complications & $17(58.6)$ \\
$\quad$ Postprocedural SAH & $13(44.8)$ \\
Distal embolization & $7(24.1)$ \\
Parenchymal ICH & $4(13.8)$ \\
$\quad$ ICH $\leq 30 \%$ of infarcted area with mild mass effect & $3(10.3)$ \\
ICH $>30 \%$ of infarcted area with notable mass effect & $4(2-5)$ \\
mRS at discharge & $9(31.0)$ \\
mRS 0-2 & $20(69.0)$ \\
mRS $\geq 3$ &
\end{tabular}

Values are presented as number (\%) or median (interquartile range). $\mathrm{mTICl}$, modified thrombolysis in cerebral infarction; $\mathrm{SAH}$, subarachnoid hemorrhage; ICH, intracerebral hemorrhage; mRS, modified Rankin scale. 
Table 5. Comparison of clinical factors (initial NIHSS, ASPECTS, grade of recanalization, and postprocedural complications) affecting the prognosis

\begin{tabular}{lccc}
\hline (\% in each group) & $m R S 0-2(n=9)$ & $m R S \geq 3(n=20)$ & P-value \\
\hline Initial NIHSS & $14.3 \pm 3.4$ & $16.9 \pm 3.5$ & 0.08 \\
Initial ASPECTS & $9.1 \pm 0.6$ & $7.6 \pm 1.2$ & $7(100.0)$ \\
$\mathrm{mTICl}$ 0-2a & 0 & $13(59.1)$ & 0.001 \\
$\mathrm{mTICl}$ 2b-3 & $9(40.9)$ & $12(70.6)$ & 0.06 \\
Postprocedural SAH & $5(29.4)$ & $7(53.8)$ & 0.8 \\
Distal embolization & $6(46.2)$ & $6(85.7)$ & 0.2 \\
Parenchymal ICH & $1(14.3)$ & 0.4
\end{tabular}

Values are presented as mean \pm standard deviation or number $(\%)$.

NIHSS, National Institute of Health Stroke Scale; ASPECTS, Alberta Stroke Programmed Early Computed Tomography Score; mRS, modified Rankin scale; mTICl, modified thrombolysis in cerebral infarction; SAH, subarachnoid hemorrhage; ICH, intracerebral hemorrhage.

Table 6. Comparison of periprocedural clinical factors between patients with and without ICA dolichoectasia

\begin{tabular}{lccc}
\hline (\% in each group) & ICA dolichoectasia $(+)(n=9)$ & ICA dolichoectasia( $(-)(n=20)$ & P-value \\
\hline Puncture to recanalization $(\min )$ & $53 \pm 43.9$ & $51.6 \pm 22$ & $>0.9$ \\
No. of passes & $2.6 / 1$ & $2.4 / 2$ & 0.9 \\
$\quad$ No. of passes $\geq 3$ & $3(33.3)$ & $8(40.0)$ & $>0.9$ \\
mTICl 0-2a & $1(11.1)$ & $6(30.0)$ & 0.4 \\
mTICl 2b-3 & $8(88.9)$ & $14(70.0)$ & \\
SAH & $5(55.6)$ & $12(60.0)$ & 0.8 \\
Distal embolization & $3(33.3)$ & $10(50.0)$ & 0.5 \\
Parenchymal ICH & $2(22.2)$ & $5(25.0)$ & $>0.9$
\end{tabular}

Values are presented as mean \pm standard deviation, mean/median, or number $(\%)$.

ICA, internal carotid artery; mTICl, modified thrombolysis in cerebral infarction; SAH, subarachnoid hemorrhage; ICH, intracerebral hemorrhage.

There was no statistical correlation, but patients with ICA dolichoectasia showed a higher tendency for good recanalization outcome as an mTICI score of $2 b-3$. Fourteen patients had an mTICI score of $2 b-3$ in 20 patients without ICA dolichoectasia. Furthermore, there was no tendency for prolongation of the mean puncture to recanalization time or the mean passage trial time in the group of patients with ICA dolichoectasia; post-procedure complications did not commonly occur (Table 6).

When comparing the correlation between ICA dolichoectasia and passage trial time, there was no tendency for more passage times needed for higher grade ICA dolichoectasia, and there was no tendency for more passage trials needed as a larger angle of CCA takeoff angle. Almost all passages were performed only in 1 to 2 times. In the distal ICA occlusion, there were more passage trial times needed compared to MCA occlusion, which is considered due to more in-situ infarction in the distal ICA occlusion case. Moreover, there was no tendency for a lower mTICI score for higher grade ICA dolichoectasia, and there was no tendency for a lower mTICI score as the angle of CCA takeoff angle increased.

\section{Discussion}

In this study, we showed recanalization of mTICI $2 b-3$ for large-vessel intracranial occlusion of the anterior circulation in 22 out of 29 patients (75.9\%), consistent with the previous studies $[6,10]$. As suggested in previous studies, the initial NIHSS, ASPECTS, and mTICI grade were significantly associated with a favorable outcome [10]. However, in contrast to previous studies, proximal tortuosity, such as ICA dolichoectasia and CCA takeoff angle, was not associated with the procedure time, the number of passages, and unfavorable outcome [5]. Furthermore, procedure-related intracranial hemorrhage was not a significant factor that affected the patients' outcomes in this study.

Although there was a limitation in obtaining statistical significance due to the small number of patients, we could confirm that there was no significant association between proximal tortuosity and the number of passes or mTICI showing the scatter plots (Fig. 2).

Advancements in endovascular devices may have contributed to the decrease in procedure-related complications. For example, improved proximal support of the balloon guiding catheter and softness of the aspiration catheter enabled the approach to the intra- 


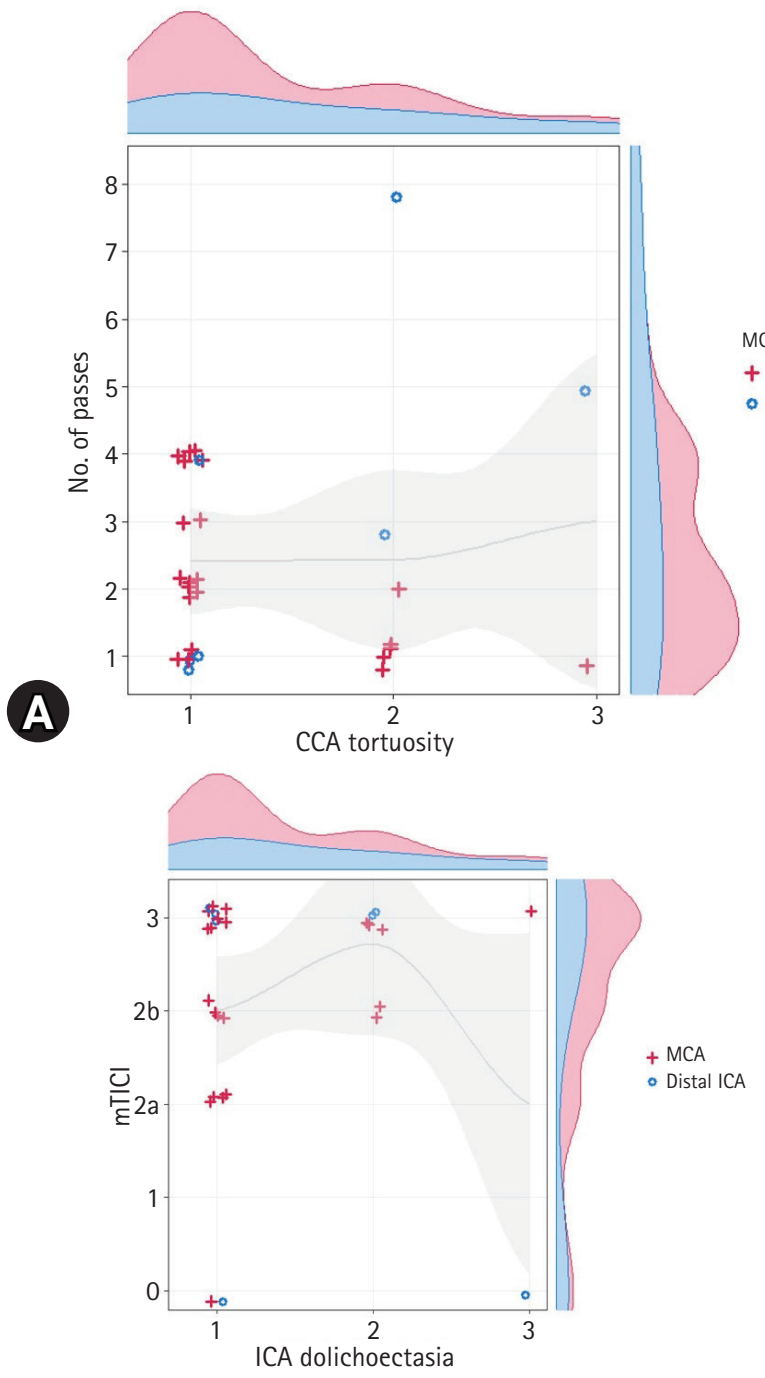

\section{c}

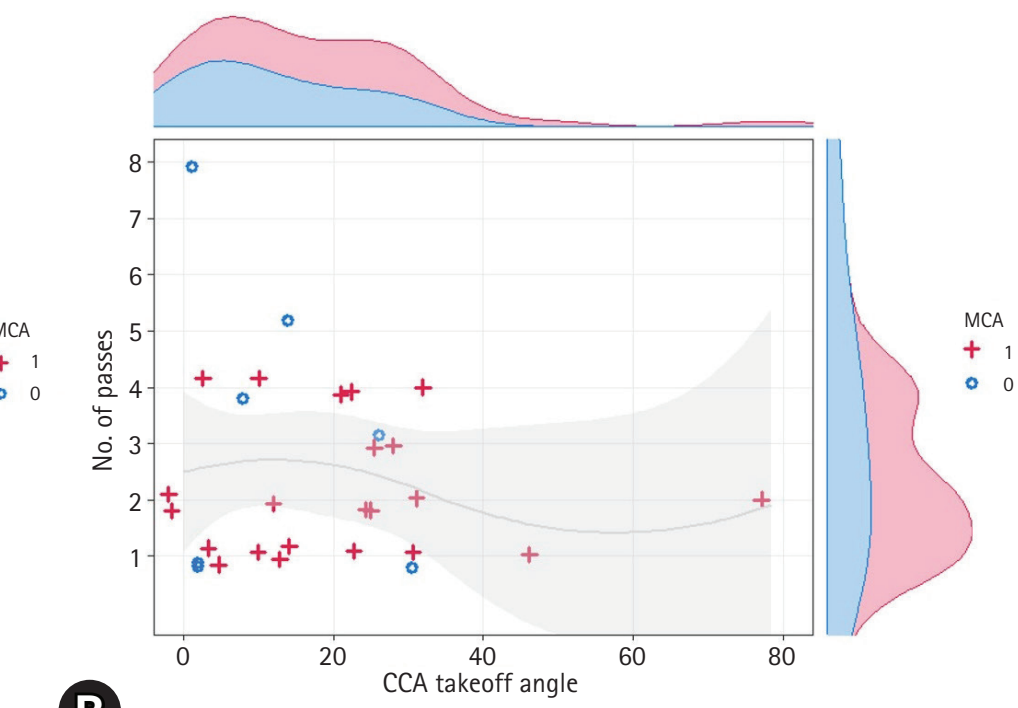

B

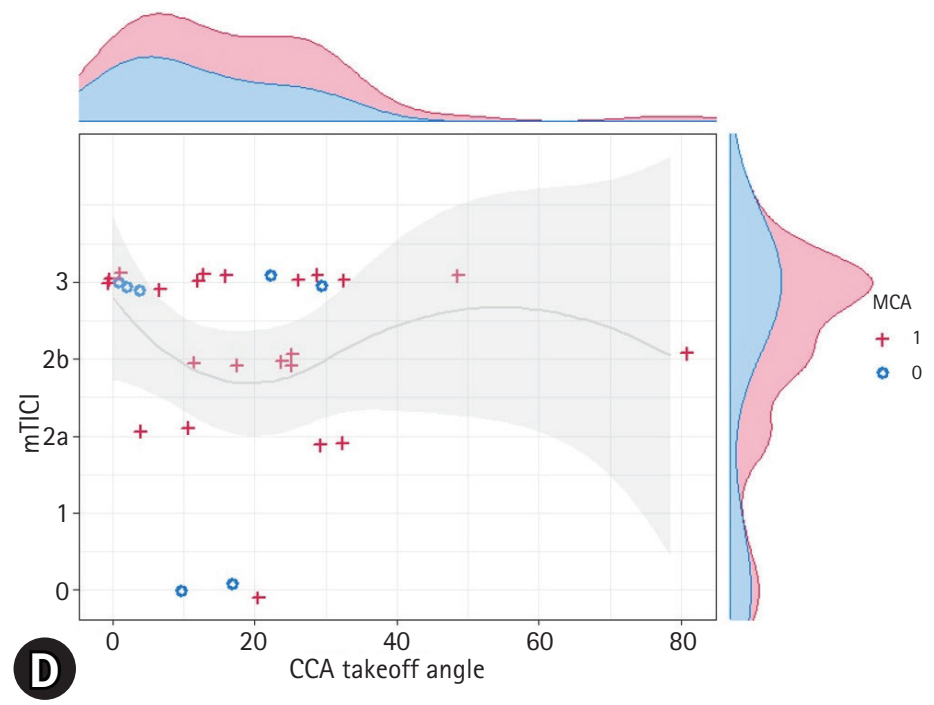

Fig. 2. Scatter plots presenting the relationship between vascular tortuosity and results after intra-arterial thrombectomy. (A) Internal carotid artery (ICA) dolichoectasia and number of passes. (B) Common carotid artery (CCA) takeoff angle and number of passes. (C) ICA dolichoectasia and modified thrombolysis in cerebral infarction (mTICl) grade. (D) CCA takeoff angle and mTICl grade. MCA, middle cerebral artery.

cranial arteries to overcome proximal tortuosity in older patients [11-13]. In addition, the combination of stent retriever method and clot-contact aspiration has contributed to the improved recanalization rate in large intracranial artery occlusion $[1,13,14]$.

The results of this study suggest that proximal tortuosity is not the main factor that affects procedural risk and patient outcome in older patients when performing mechanical IAT. Moreover, post-procedural SAH and distal embolism, which may be related to the procedures, were not the main factors associated with unfavorable outcomes for patients.

Therefore, we consider that IAT may be more widely indicated in older patients with large intracranial artery occlusion owing to the advancement of endovascular devices and techniques compared to the past.

\section{Limitations of the study}

Due to the limited number of patients and the retrospective nature of the study, this study has the limitation of reaching a concrete conclusion in deciding IAT in older patients. However, this study was unique because we showed equivalent clinical outcomes after IAT in older patients compared to previous studies with all age groups. 
A prospective study with a larger number of patients by comparing different techniques would be necessary for future studies. Moreover, this study was limited to patients with ischemic stroke in the anterior circulation. Further evaluation of clinical outcomes is warranted in patients with posterior circulation occlusion.

\section{Conclusion}

There was no correlation between the severity of ICA dolichoectasia and the increase in the number of passages and the tendency for poor recanalization after IAT. In addition, there was no correlation between the severity of the CCA takeoff angle and the increase in the number of passages and the tendency for poor recanalization. We can clarify that the limitation of the procedure based on tortuosity of vessels in older patients is not justifiable.

\section{Conflicts of interest}

No potential conflict of interest relevant to this article was reported.

\section{Acknowledgments}

This research was supported by the Department of Neurosurgery, Hallym University Sacred Heart Hospital.

\section{ORCID}

Su Min Kye, https://orcid.org/0000-0002-4448-3046

Jun Hyong Ahn, https://orcid.org/0000-0002-8529-6757

In Bok Chang, https://orcid.org/0000-0001-8003-3264

Joon Ho Song, https: / / orcid.org/0000-0003-3138-074X

Ji Hee Kim, https://orcid.org/0000-0001-6937-8093

Heui Seung Lee, https://orcid.org/0000-0002-4075-710X

Jae Keun Oh, https://orcid.org/0000-0001-5127-2661

\section{REFERENCES}

1. Campbell BC, Hill MD, Rubiera M, et al. Safety and efficacy of solitaire stent thrombectomy: individual patient data meta-analysis of randomized trials. Stroke 2016;47:798-806.

2. Jovin TG, Chamorro A, Cobo E, et al. Thrombectomy within 8 hours after symptom onset in ischemic stroke. N Engl J Med 2015;372:2296-306.
3. Kurre W, Aguilar-Pérez M, Niehaus L, et al. Predictors of outcome after mechanical thrombectomy for anterior circulation large vessel occlusion in patients aged $\geq 80$ years. Cerebrovasc Dis 2013;36:430-6.

4. Snelling BM, Sur S, Shah SS, et al. Unfavorable vascular anatomy is associated with increased revascularization time and worse outcome in anterior circulation thrombectomy. World Neurosurg 2018; 120:e976-83.

5. Singer OC, Haring HP, Trenkler J, et al. Age dependency of successful recanalization in anterior circulation stroke: the ENDOSTROKE study. Cerebrovasc Dis 2013;36:437-45.

6. Azkune Calle I, Bocos Portillo J, Anton-Ladislao A, et al. Clinical outcome of mechanical thrombectomy for stroke in the elderly.J Stroke Cerebrovasc Dis 2017;26:582-8.

7. Alawieh A, Chatterjee A, Feng W, et al. Thrombectomy for acute ischemic stroke in the elderly: a 'real world' experience. J Neurointerv Surg 2018; 10:1209-17.

8. Alawieh A, Starke RM, Chatterjee AR, et al. Outcomes of endovascular thrombectomy in the elderly: a 'real-world' multicenter study. J Neurointerv Surg 2019;11:545-53.

9. Knox JA, Alexander MD, McCoy DB, et al. Impact of aortic arch anatomy on technical performance and clinical outcomes in patients with acute ischemic stroke. AJNR Am J Neuroradiol 2020;41:268-73.

10. Barral M, Lassalle L, Dargazanli C, et al. Predictors of favorable outcome after mechanical thrombectomy for anterior circulation acute ischemic stroke in octogenarians. J Neuroradiol 2018; 45:211-6.

11. Son S, Kang DH, Hwang YH, Kim YS, Kim YW. Efficacy, safety, and clinical outcome of modern mechanical thrombectomy in elderly patients with acute ischemic stroke. Acta Neurochir (Wien) 2017;159:1663-9.

12. Chueh JY, Kang DH, Kim BM, Gounis MJ. Role of balloon guide catheter in modern endovascular thrombectomy.J Korean Neurosurg Soc 2020;63:14-25.

13. Kang DH, Park J. Endovascular stroke therapy focused on stent retriever thrombectomy and direct clot aspiration: historical review and modern application. J Korean Neurosurg Soc 2017; 60:335-47.

14. Turk AS 3rd, Siddiqui A, Fifi JT, et al. Aspiration thrombectomy versus stent retriever thrombectomy as first-line approach for large vessel occlusion (COMPASS): a multicentre, randomised, open label, blinded outcome, non-inferiority trial. Lancet 2019; 393:998-1008. 